Elsevier required licence: (C) <2018>. This manuscript version is made available under the CC-BY-NCND 4.0 license http://creativecommons.org/licenses/by-nc-nd/4.0/

The definitive publisher version is available online at

[https://www.sciencedirect.com/science/article/pii/S0016003218303399?via\%3Dihub] 


\title{
Sparsely distributed sliding mode control for interconnected systems
}

\author{
AHMADREZA ARGHA \\ School of Electrical Engineering and Telecommunications, University of New South Wales, Sydney, NSW 2052, Australia
}

STEVEN W. SU*

\section{Li LI, AND HUNG NGUYEN}

Faculty of Engineering and Information Technology, University of Technology Sydney, PO Box 123, Broadway, NSW 2007, Australia

May 8, 2019

\begin{abstract}
This paper proposes a framework for the design of sparsely distributed output feedback discrete-time sliding mode control (ODSMC) for interconnected systems. The major target here is to develop an observer based discrete-time sliding mode controller employing a sparsely distributed control network structure in which local controllers exploit some other sub-systems' information as well as its own local information. As the local controllers/observers have access to some other sub-systems' states, the control performance will be improved and the applicability region will be widened compared to the decentralised structure. As the first step, a stability condition is derived for the overall closed-loop system obtained from applying ODSMC to the underlying interconnected system, by assuming a priori known structure for the control/observer network. The developed LMI based controller design scheme provides the possibility to employ different information patterns such as fully distributed, sparsely distributed and decentralised patterns. In the second step, we propose a methodology to identify a sparse control/observer network structure with the least possible number of communication links that satisfies the stability condition given in the first step. The boundedness of the obtained overall closed-loop system is analysed and a bound is derived for the augmented system state which includes the closed-loop system state and the switching function.
\end{abstract}

\section{INTRODUCTION}

The modern practical systems such as power distribution networks, water distribution systems, manufacturing systems, biological systems, computer communication systems, irrigation systems and transportation systems can be considered as large-scale interconnected dynamical systems, for which decentralised and distributed control schemes have been proposed. Utilising a centralised control scheme in networked control systems (NCSs), which requires the central controller to have access to the states of all subsystems' plants, is not practical as it needs a larger and more costly control network. The main idea behind the decentralised control scheme is to use only the local state information in order to control the subsystems and thus there is no control network. This can be effective only when the interconnections between the subsystems are not strong $[1,2]$. When the interconnections are strong, utilising distributed control frameworks has been considered. In this strategy, each subsystem can exploit local states as well as some of the other subsystems states. Hence, compared to the decentralised control scheme, distributed control scheme can ensure the stability of large scale interconnected systems in the presence of stronger subsystem interconnections [3]. Meantime, it also has less complexity and improved computational aspects compared to the centralised control scheme. The idea of spatially distributed control systems is implementable by using NCSs. In NCSs, local measurements are transferred among local controllers/observers using a control network. Spatially distributed control systems have extensive applications such as electrical networks, factory automation and transportation networks [4].

In interconnected systems, the structure of the distributed controller network is usually restricted due to a number of factors such as implementation-related concerns and communication costs. This issue in distributed systems is also referred to as information pattern, which means that, unlike traditional distributed control schemes in which all the involving sub-controllers share the same information, the sub-controllers can share or receive different information [5]. Since the

*Corresponding author, steven.su@uts.edu.au 
fully distributed controller structure is not always feasible, one may consider the design of distributed control systems with imposing a priori constraints on communication network structure. Alternatively, another choice for the distributed control systems is to design a control network with the minimum number of communication links while satisfying a global control objective $[4,6]$. Indeed, a trade off between the control performance and sparsity of the control network should be considered [7, 8,9]. Note that recently several work in the literature has focused on the design of sparsely distributed controller/observer networks; see e.g. [4, 7]. Indeed, this is performed for minimising the overall cost of the control network utilised to control the system. Assuming that the general cost, including the construction and data transferring costs etc, are identical for all the links, minimising the costs of a control network can intuitively be considered as minimising the number of communication links in the control network structure or equivalently finding the sparsest control network structure satisfying the control objective.

Basically, to address network sparsification problems, one has to, in worst case, check all possible topologies, implying an exhaustive search for a number of configurations that can grow exponentially with the number of communication links. This is practically intractable and impossible to perform. As explained in [10], to avoid performing an exhaustive search, a trade-off can be made either in the choice of the search strategy or in the choice of the selection criterion. Another alternative to avoid solving a combinatorial problem is to consider a multi-objective problem of controller structure and control law co-design by incorporating secondary cost functions which are convex approximations of the original $\ell_{0}$-quasi-norms and can promote sparsity of the distributed controller, into a main cost function, which represents a performance specification of the closed-loop system [7, 11]. The reweighted $\ell_{1}$ (REL1) norm algorithms can be further employed to identify the sparse optimal feedback gain [7]. The weights (entries of the weighting matrix), in the REL1 algorithms, are updated at each iteration using the solutions of the previous iteration.

One thread of the literature of sliding mode control (SMC) has focused on developing decentralised SMC for the interconnected systems; see $[12,13,14,15]$ and the references therein. Nevertheless, the distributed SMC has received less attention and hence it requires more investigations. The objective of this paper is to extend the idea of spatially distributed control systems to the field of discrete-time SMC (DSMC) problems. This paper firstly explores the problem of designing a sparse DSMC network with an arbitrary but fixed topology for a given networked system. A methodology is provided to stabilise the underlying interconnected dynamics utilising a (sparsely) distributed controller/observer network. We will show that the proposed observer-based DSMC has the ability to cover all the cases such as fully decentralised, fully distributed, and sparsely distributed topologies. It is worth noting that exploiting a sparse structure for the control network, which is a subset of dynamics structure, is crucial in the control system for large scale systems, for instance, the smart grids [16]. The next arising problem is how to find a sparse control network structure that satisfies the control objective. One may resort to find the sparsest control/observer structure that can stabilise the interconnected system. This issue has been investigated in e.g. [7] in order to find the suboptimal controllers that minimise a special objective function considering the sparsity of the feedback gain. However, this may result in a non-convex condition. Also, the authors of [4] have considered the problem of finding the sparsest control/observer network that satisfies a set of stability conditions, obtained through a Lyapunov direct scheme. In this paper, as the second step, we will search for a sparse control/observer network structure with the least possible number of communication links satisfying the given stability condition. To this end, a heuristic iterative algorithm will be proposed, distinguishing itself from a trial-and-error process which requires to check all the possible structures.

Disturbance observer-based control strategies have been exploited in different fields in the literature; see e.g. [17]. The authors of [18] have proposed to employ disturbance estimate in the DSMC rather than a discontinuous component. It is also stated that this can reduce the boundary layer thickness. However, the DSMC in [18] is only applicable to the problems whose system states are available and more importantly no unmatched uncertainty exists. The method in [19] exploits only output information for discrete-time MIMO systems involving unmatched exogenous disturbances but without unmatched uncertainties, by using the so-called proportional integral observer. The distributed output feedback DSMC (ODSMC), presented in this paper, utilises a disturbance observer in order to deal with the influences of the exogenous disturbances on the boundary layer thickness. This sparsely distributed ODSMC is designed by means of an LMI scheme.

In brief, the main contributions of this paper are on

- designing a sparse discrete-time sliding mode control (DSMC) network with an arbitrary but fixed topology for a given networked system;

- providing a methodology for stabilising the underlying interconnected dynamics utilising a (sparsely) distributed controller/observer network;

- searching for a sparse control/observer network structure with the least possible number of communication links satisfying the given stability condition.

This paper includes the following major novel folds:

- In contrast to the current methods for the design of sparse feedback gains for NCSs in most existing literature assuming all the system states are available, this paper proposes a sparse DSMC scheme to control the system by directly employing the measured system outputs. 
- A general explicit framework for the design of sparse or structured feedback gains is developed which can address the structured and sparse output feedback DSMC controller design problem for uncertain NCSs.

- The proposed observer-based DSMC has the ability to cover all the cases such as fully decentralised, fully distributed, and sparsely distributed topologies.

- A method for exploring a sparse control/observer network structure with the least possible number of communication links is proposed which can satisfy the given stability condition.

Notation: $\left[\Sigma_{i j}\right]_{r \times r}$ is a block matrix with block entries $\Sigma_{i j}, i=1, \cdots, r, j=1, \cdots, r$. diag $\left[\Sigma_{i i}\right]_{i=1}^{r}$ is a block-diagonal matrix with block entries $\Sigma_{i i}, i=1, \cdots, r$. Moreover, $\operatorname{col}\left(v_{i}(k)\right)_{i=1}^{r}$ denotes a block-vector with block diagonal entries $v_{i}(k), i=1, \cdots, r .\{0\}$ denotes an operator for $\Xi=\left[\xi_{i j}\right]_{h \times h}$ in which $\xi_{i j} \in \mathbb{R}$ and $W=\left[W_{i j}\right]_{h \times h}$ in which $W_{i j} \in \mathbb{R}^{r_{i} \times s_{j}}$ such that $\Xi \circ W=\left[\xi_{i j} W_{i j}\right]_{h \times h}$. $\otimes$ denotes the Kronecker product.

\section{PROBLEM Formulation AND PRELIMINARIES}

Consider an interconnected system consisting of $h$ sub-systems:

$$
\left\{\begin{array}{l}
x_{i}(k+1)=\left[G_{i i}+\Delta G_{i i}(k)\right] x_{i}(k)+H_{i}\left[u_{i}(k)+\xi_{i}(k)\right]+\sum_{j=1, j \neq i}^{h}\left[G_{i j}+\Delta G_{i j}(k)\right] x_{j}(k), \\
y_{i}(k)=C_{i} x_{i}(k), \quad i=1, \cdots, h,
\end{array}\right.
$$

where $x_{i} \in \mathbb{R}^{n_{i}}, y_{i} \in \mathbb{R}^{p_{i}}$ and $u_{i} \in \mathbb{R}^{m_{i}}$ are the state vector, output vector and control input vector of the $i$-th sub-system, respectively. Without loss of generality, it is assumed that $m_{i} \leq p_{i} \leq n_{i}, \operatorname{rank}\left(B_{i}\right)=m_{i}, \operatorname{rank}\left(C_{i}\right)=p_{i}$. The term $\Delta G_{i i}(k)$ denotes the uncertainty of $i$-th sub-system and $\sum_{\substack{j=1 \\ j \neq i}}^{h} G_{i j} x_{j}(k), \sum_{\substack{j=1 \\ j \neq i}}^{h} \Delta G_{i j}(k) x_{j}(k)$ are, respectively, a known interconnection and an uncertain interconnection of the $i$-th sub-system. We also assume

$$
\Delta G_{i j}(k)=\Xi_{i j} \Theta_{i j}(k) \Lambda_{i j},
$$

where $\Xi_{i j}$ and $\Lambda_{i j}$ are known matrices and $\Theta_{i j}(k)$ is an unknown time varying matrix satisfying $\Theta_{i j}^{T}(k) \Theta_{i j}(k) \leq I . \xi_{i}(k)$ is the matched external disturbances of the $i$-th sub-system with a known bound.

Remark 1. It is worth noting that the assumption on the norm boundedness of $\xi_{i}(k)$ is a very common assumption in several fields of control theory, especially SMC. Alternatively, the assumption that the disturbance is given by an additive random noise with (partially) known probability density function (pdf) can be made. However, in many real cases disturbance may not be of the very random nature. An interesting approach, which has progressively been employed in the literature, is set membership or unknown but bounded (UBB) error description [20].

In order to make the problem closer to practical cases and improve the generality of the controller synthesis problem, we consider mismatched uncertainties, i.e., $\Delta G_{i i}$ and $\Delta G_{i j}$ as shown in the system (1).

Define

$$
\begin{aligned}
& x(k):=\operatorname{col}\left(x_{i}(k)\right)_{i=1}^{h}, u(k):=\operatorname{col}\left(u_{i}(k)\right)_{i=1}^{h}, \\
& y(k):=\operatorname{col}\left(y_{i}(k)\right)_{i=1}^{h}, \xi(k):=\operatorname{col}\left(\xi_{i}(k)\right)_{i=1}^{h},
\end{aligned}
$$

and

$$
\begin{aligned}
G & :=\left[G_{i j}\right]_{h \times h}, \Delta G(k):=\left[\Delta G_{i j}(k)\right]_{h \times h}, \\
H & :=\operatorname{diag}\left[H_{i}\right]_{i=1}^{h}, C:=\operatorname{diag}\left[C_{i}\right]_{i=1}^{h} .
\end{aligned}
$$

Also, the uncertainty matrix for overall system can be rearranged as:

$$
\Delta G(k)=\sum_{i=1}^{h} \Xi_{i} \Theta_{i}(k) \Lambda_{i},
$$

in which

$$
\begin{aligned}
\Xi_{i} & =\left(e_{i} \otimes I\right)\left[\begin{array}{lll}
\Xi_{i 1} & \cdots & \Xi_{i h}
\end{array}\right], \\
\Theta_{i}(k) & =\operatorname{diag}\left(\Theta_{i 1}(k), \cdots, \Theta_{i h}(k)\right), \\
\Lambda_{i} & =\operatorname{diag}\left(\Lambda_{i 1}, \cdots, \Lambda_{i h}\right),
\end{aligned}
$$

with $e_{i}$ denoting the canonical basis of $\mathbb{R}^{h}$ and $\otimes$ being the Kronecker product. Using (1), (3) and (4), the system in (1) at the network level can be written as:

$$
\left\{\begin{array}{l}
x(k+1)=[G+\Delta G(k)] x(k)+H[u(k)+\xi(k)] \\
y(k)=C x(k) .
\end{array}\right.
$$




\section{i. Preliminaries}

Definition 1. A matrix whose elements are either 0 or 1 is said to be a structure matrix. Let $\Lambda=\left[\Lambda_{i j}\right]_{m \times n}$ be a block matrix with $\Lambda_{i j} \in \mathbb{R}^{a_{i} \times b_{j}}$, then the structure matrix of $\Lambda$ is obtained as $S(\Lambda) \triangleq\left[\gamma_{i j}\right]_{m \times n}$ with

$$
\gamma_{i j}= \begin{cases}0 & \text { if } \Lambda_{i j}=0, i \neq j, \\ 1 & \text { otherwise. }\end{cases}
$$

Definition 2. Two matrices $\Lambda_{1}$ and $\Lambda_{2}$ are structurally the same if $S\left(\Lambda_{1}\right)=S\left(\Lambda_{2}\right)$.

Definition 3. The matrix $\Lambda_{1}$ with $\mathrm{S}\left(\Lambda_{1}\right) \triangleq\left[\gamma_{i j}^{1}\right]_{m \times n}$ is structurally a subset of $\Lambda_{2}$ with $\mathrm{S}\left(\Lambda_{2}\right) \triangleq\left[\gamma_{i j}^{2}\right]_{m \times n}$ if $\gamma_{i j}^{2}-\gamma_{i j}^{1} \geq 0$. We denote this as $\mathrm{S}\left(\Lambda_{1}\right) \subseteq \mathrm{S}\left(\Lambda_{2}\right)$.

Lemma 1. Consider

$$
0<W=\left[\begin{array}{ll}
\operatorname{diag}\left[\bar{W}_{i}\right]_{i=1}^{h} & \operatorname{diag}\left[\hat{W}_{i}\right]_{i=1}^{h} \\
\operatorname{diag}\left[\hat{W}_{i}\right]_{i=1}^{h} & \operatorname{diag}\left[\hat{W}_{i}\right]_{i=1}^{h}
\end{array}\right] \in \mathbb{R}^{(m+n) \times(m+n)},
$$

with $\bar{W}_{i} \in \mathbb{R}^{n_{i} \times n_{i}}, \tilde{W}_{i} \in \mathbb{R}^{m_{i} \times m_{i}}, \hat{W}_{i} \in \mathbb{R}^{n_{i} \times m_{i}}$. We have

$$
\mathrm{S}(W)=\mathrm{S}\left(W^{-1}\right) .
$$

Proof. This lemma can easily be proved by applying the block matrix inverse formula.

Lemma 2. Consider the matrix $0<W \in \mathbb{R}^{(m+n) \times(m+n)}$ given in Lemma 1 and let $\Gamma$ be a known structure matrix. For any $Y=\left[\begin{array}{l}Y_{1} \\ Y_{2}\end{array}\right]$, where $Y_{1} \in \mathbb{R}^{n \times p}$ and $Y_{2} \in \mathbb{R}^{m \times p}$, while $\left[\begin{array}{l}J_{1} \\ J_{2}\end{array}\right]=W^{-1} Y$ and $\mathrm{S}\left(Y_{1}\right) \subseteq \Gamma, \mathrm{S}\left(Y_{2}\right) \subseteq \Gamma$, we have

$$
\begin{aligned}
& \mathrm{S}\left(J_{1}\right) \subseteq \Gamma, \\
& \mathrm{S}\left(J_{2}\right) \subseteq \Gamma .
\end{aligned}
$$

Proof. Due to the simplicity, we omit the proof here.

Although the proofs of Lemmas 1, 2 are straightforward, these lemmas can be applied to relax the structure limitation from block diagonal to $\mathrm{S}(W)$, which tolerates the off-diagonal blocks given that they are also in block diagonal forms. To the best knowledge of the authors, these lemmas are new in literature.

Definition 4. The overall system (6) is said to be structurally controllable with respect to the structure matrix $\Gamma=\left[\gamma_{i j}\right]_{h \times h}$ if there exists $K=\left[K_{i j}\right]_{h \times h}$ with $\mathrm{S}(K) \subseteq \Gamma$ such that the modes of $G-H K$ are arbitrarily assignable.

Definition 5. The overall system (6) is said to be structurally observable with respect to the structure matrix $\Gamma=\left[\gamma_{i j}\right]_{h \times h}$ if there exists $L=\left[L_{i j}\right]_{h \times h}$ with $\mathrm{S}(L) \subseteq \Gamma$ such that the modes of $\Gamma \circ G-L C$ are arbitrarily assignable, where $\Gamma \circ G=$ $\left[\gamma_{i j} G_{i j}\right]_{h \times h}$.

Assumption 1. The matrix triple $(G, H, C)$ in (6) is structurally controllable and observable with respect to the given structure matrix $\Gamma=\left[\gamma_{i j}\right]_{h \times h}$.

Assumption 2 ([19]). The matrices $G, H$ and $C$ in the system (6) and the structure matrix $\Gamma$ satisfy

$$
\operatorname{rank}\left(\left[\begin{array}{cc}
\Gamma \circ G-I_{n} & H \\
C & 0
\end{array}\right]\right)=n+m .
$$

Note that the above assumption is equivalent to not having transmission zero at 1.

Assumption 3. The exogenous disturbance $\xi_{i}(k)$ in the i-th sub-system in (1) satisfies the Lipschitz continuity condition:

$$
\left\|\tilde{\xi}_{i}(k)\right\| \leq \tilde{L}_{i} T_{s}, \quad \forall k \geq 0,
$$

where $\tilde{\xi}_{i}(k)=\xi_{i}(k)-\xi_{i}(k-1), \tilde{L}_{i}>0$ denotes the Lipschitz constant and $T_{s}$ is the sampling time.

The above assumption is a usual assumption in the control theory literature, specifically when using the disturbance estimate in the control law is the case; see [18]. We assume that $\tilde{L}_{i}$ is a small scalar. For this reason, we assume that the sampling rate of the discrete signal processing system is large enough compared to the maximum frequency component of the exogenous disturbance $\xi_{i}(k)$. 


\section{ii. State and disturbance observer}

In this paper, the estimation scheme below is proposed to provide the $i$-th local controller with the system state information and disturbance estimate,

$$
\left\{\begin{array}{l}
\hat{x}_{i}(k+1)=G_{i i} \hat{x}_{i}(k)+H_{i} u_{i}(k)+\sum_{j=1, j \neq i}^{j=h} \gamma_{i j} G_{i j} \hat{x}_{j}(k)+\sum_{j=1}^{j=h} \gamma_{i j} L_{i j}\left[y_{j}(k)-\hat{y}_{j}(k)\right]+H_{i} \hat{\xi}_{i}(k) \\
\hat{\xi}_{i}(k+1)=\hat{\xi}_{i}(k)+\sum_{j=1}^{j=h} \gamma_{i j} D_{i j}\left[y_{j}(k)-\hat{y}_{j}(k)\right] \\
\hat{y}_{i}(k)=C_{i} \hat{x}_{i}(k),
\end{array}\right.
$$

where $\hat{x}_{i}(k) \in \mathbb{R}^{n_{i}}$ is the state estimate of the $i$-th sub-system in (1), $\hat{y}_{i}(k) \in \mathbb{R}^{p_{i}}$ is the observer output, $L_{i j} \in \mathbb{R}^{n_{i} \times p_{j}}$ and $D_{i j} \in \mathbb{R}^{m_{i} \times p_{j}}$ are local observer gains if $i=j$ and coupling observer gains if $i \neq j$ for the state and disturbance respectively. Here $\gamma_{i j}$ denotes the availability of communication links among subsystems in the controller and observer design, that is, $\gamma_{i j}=1$ if $i j$-th link exists in the control/observer network and $\gamma_{i j}=0$ otherwise. Then the overall estimator is

$$
\left\{\begin{array}{l}
\hat{x}(k+1)=\Gamma \circ G \hat{x}(k)+H u(k)+L_{s}[y(k)-\hat{y}(k)]+H \hat{\xi}(k) \\
\hat{\xi}(k+1)=\hat{\xi}(k)+D_{s}[y(k)-\hat{y}(k)] \\
\hat{y}(k)=C \hat{x}(k)
\end{array}\right.
$$

where $L_{s}:=\Gamma \circ L$ with $L=\left[L_{i j}\right]_{h \times h}, D_{s}:=\Gamma \circ D$ with $D=\left[D_{i j}\right]_{h \times h}$ and $\Gamma=\left[\gamma_{i j}\right]_{h \times h}$.

\section{Spatially Decentralised Sliding Mode Control}

The goal of this paper is to determine an appropriate decentralised sliding surface as:

$$
\mathscr{S}:=\{x(k) \mid \sigma(k) \triangleq S x(k)=0\},
$$

where $\sigma:=\operatorname{col}\left(\sigma_{i}\right)_{i=1}^{h}$ and $S:=\operatorname{diag}\left[S_{i}\right]_{i=1}^{h}$ is a block diagonal matrix which will be designed in the sequel of this section such that $\mathrm{SH}$ is invertible, and a distributed control law which depends only on the system outputs such that:

- in the absence of external matched disturbance $(\xi=0)$ and in the presence of mismatched uncertainty $(\Delta G)$, an ideal sliding motion is achieved in finite time;

- in the presence of external matched disturbance and mismatched uncertainty, the harmful influence of the matched disturbance is minimised and an appropriate bounded motion around the ideal decentralised sliding surface $\mathscr{S}$ is maintained.

One may obtain from (6) and (10) that

$$
\sigma(k+1)=S[G+\Delta G] x(k)+S H[u(k)+\xi(k)] .
$$

Remark 2. Notice that since the sliding function $\sigma(\cdot)$ will not be used in the variable structure discontinuous component of the ODSMC, the decentralised sliding surface is not required to be designed by utilising known information of the system. Instead, it is only required to be ensured that the system state trajectories are driven into a boundary layer about the decentralised sliding surface and be kept there thereafter. This is the key feature of the ODSMC presented in this paper for NCSs. This can also lead to a considerable extension to the applicable region of the framework given in this paper compared to the existing literature for the continuous-time counterpart. The same manner can be seen in [21] for the static ODSMC.

The distributed controller is assumed to have the following form:

$$
u_{i}(k)=-\left(S_{i} H_{i}\right)^{-1}\left[\left(S_{i} G_{i i}-\Phi_{i} S_{i}\right) \hat{x}_{i}(k)+S_{i} \sum_{j=1, j \neq i}^{j=h} \gamma_{i j} G_{i j} \hat{x}_{j}(k)\right]-\hat{\xi}_{i}(k)
$$

where $\Phi_{i} \in \mathbb{R}^{m_{i} \times m_{i}}$ is a stable matrix and aims to govern the state convergence rate. Here, similar to [22], it is assumed that $\Phi_{i}=\lambda_{i} I_{m_{i}}$, where $0 \leq \lambda_{i}<1$ is a given constant value. The control law $u_{i}(k)$ in (12) can be written as

$$
u_{i}(k)=-\left(S_{i} H_{i}\right)^{-1} S_{i}\left[G_{i i}^{\lambda_{i}} \hat{x}_{i}(k)+\sum_{j=1, j \neq i}^{j=h} \gamma_{i j} G_{i j} \hat{x}_{j}(k)\right]-\hat{\xi}_{i}(k),
$$


where $G_{i i}^{\lambda_{i}}=G_{i i}-\lambda_{i} I_{n_{i}}$. Then the compact control law is

$$
u(k)=-(S H)^{-1} S\left(\Gamma \circ G_{\lambda}\right) \hat{x}(k)-\hat{\xi}(k),
$$

where $G_{\lambda}=G-\operatorname{diag}\left[\lambda_{i} I_{n_{i}}\right]_{i=1}^{h}$. It is worth mentioning that, referring to e.g. [21, 18, 19], the DSMC does not necessarily require switching component and the linear part in (14) leads to a boundary layer with thickness $O\left(T_{S}\right)$. As discussed in [19], by exploiting a proper sampling period in the digital processing system along with a disturbance observer in the discrete-time sliding mode control design, rather than conventional discontinuous components, the boundary layer thickness can be reduced to $O\left(T_{s}^{2}\right)$. Note also that in a previously published paper [23], we have discussed and compared different choices that can be used rather than a discontinuous component in DSMC. As the major focus of this paper is on the design of sparsely distributed DSMC, we use only the choice of disturbance estimate in the control law without any further discussion on other possible choices. We however encourage the readers to see [23]. Moreover, the controller in (14) is indeed based on the equivalent control, by removing unknown uncertainty terms and taking into account the structure constraint. The removed terms can be taken care of by robust control techniques.

Remark 3. Employing different structure matrices $\Gamma$ in the controller/observer in (14), (9), one can design different control network topologies. By letting $\gamma_{i j}=\left\{\begin{array}{ll}1 & \text { if } i=j \\ 0 & \text { otherwise }\end{array}\right.$, the decentralised control strategy will be obtained, i.e. no control network is required in the system. Additionally, if we set $\Gamma=\mathrm{S}(A)$ a fully distributed control system is derived, where each local controller exploits the states of all other physically coupled subsystems in addition to its own state. Lastly, any other arbitrary structure matrix $\Gamma \subseteq \mathscr{S}(G)$ would give a middle-of-the-road solution, between fully distributed control approaches and decentralised ones, referred to as sparsely distributed control systems.

Define the overall state estimation error as

$$
e(k):=x(k)-\hat{x}(k)
$$

and disturbance estimation error as

$$
e_{\xi}(k):=\xi(k)-\hat{\xi}(k) .
$$

Applying the controller (14) to (6) and using (15), (16) and (9), the overall closed-loop system is obtained as follows:

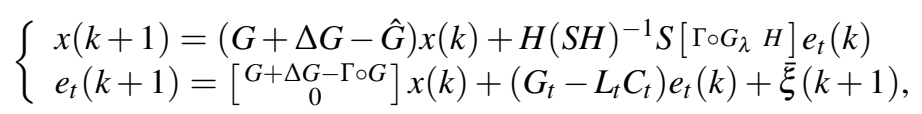

where $\hat{G}=H(S H)^{-1} S\left(\Gamma \circ G_{\lambda}\right), \bar{\xi}(k+1)=\left[\begin{array}{c}0 \\ \xi(k+1)\end{array}\right]$ with $\tilde{\xi}(k)=\operatorname{col}\left(\tilde{\xi}_{i}(k)\right)_{i=1}^{h}, e_{t}(k)=\left[\begin{array}{c}e(k) \\ e \xi(k)\end{array}\right], G_{t}=\left[\begin{array}{cc}\Gamma \circ G & H \\ 0 & I_{m}\end{array}\right]$ with $m=\sum_{i=1}^{h} m_{i}, L_{t}=\left[\begin{array}{c}L_{s} \\ D_{s}\end{array}\right]$ and $C_{t}=\left[\begin{array}{ll}C & 0\end{array}\right]$.

Lemma 3 ([19]). If the matrix pair $(\Gamma \circ G, C)$ is observable and $(\Gamma \circ G, H, C)$ satisfies the rank condition in Assumption 2 , then the matrix pair $\left(G_{t}, C_{t}\right)$ is observable.

Also, it can simply be found that

$$
\sigma(k+1)=S\left(\Delta G+G-\Gamma \circ G_{\lambda}\right) x(k)+S\left[\Gamma \circ G_{\lambda} H\right] e_{t}(k) .
$$

\section{Stability AnAlysis}

Continuous time SMC can ensure the robustness of the closed-loop system against matched disturbances and uncertainties, i.e. if the SMC is designed appropriately, the sliding motion when constrained to the switching surface is fully insensitive to matched uncertainty. However, it has been shown that its discrete time counterpart can only ensure the state trajectories to be driven into a boundary layer around the ideal sliding surface $\sigma(k)=0$ [21]. This phenomenon is referred to as quasi sliding mode (QSM) in the related literature. This is because the control command in discrete time control systems is held constant during the sample period. As a result, switching at infinite frequency is not possible, and thus, the invariance properties of continuous time SMC cannot be repeated in discrete time cases. The theorem below analyses simultaneously the reachabiltiy of QSM and the stability of the system states. For this purpose, we temporarily neglect the exogenous disturbance in the system, and only analyse the stability of the overall system, albeit with a priori known structure matrix $\Gamma$. We later characterise the boundedness of the overall closed-loop system states and sliding function's boundary layer in Theorem 2 in the sequel of this section. Moreover, it is worth noting that, since Theorem 2 will use the cross terms between the system state (sliding function) and the component $\bar{\xi}(k+1)$, the proof of Theorem 1 is started more generally (i.e. $\xi \neq 0$ and $\hat{\xi} \neq 0)$. This would help us to avoid unnecessary repetition of the technical manipulations. Letting $\xi(k)=0$, $\widetilde{\xi}(k)=0$, and thus, $\bar{\xi}(k+1)=0$, we then derive an LMI sufficient condition while ensuring the stability of the overall closed loop system. The achieved LMI is employed for the control/observer synthesis purposes. 
Theorem 1. In the absence of exogenous disturbance, i.e. $\xi(k)=0$, the linear part of the control law (14) can steer the system state onto the ideal sliding surface (10), and the system state is stabilised, if there exist matrices $P=\operatorname{diag}\left[P_{i}\right]_{i=1}^{h}$, with $0<P_{i}:=U_{i}^{T}\left[\begin{array}{cc}P_{i_{11}} & 0 \\ 0 & P_{i_{22}}\end{array}\right] U_{i}, P_{i} \in \mathbb{R}^{n_{i} \times n_{i}}, Q=\left[\begin{array}{c}\operatorname{diag}\left[\bar{Q}_{i}\right]_{i=1}^{h} \operatorname{diag}\left[\hat{Q}_{i}\right]_{i=1}^{h} \\ \operatorname{diag}\left[\hat{Q}_{i}\right]_{i=1}^{h} \operatorname{diag}\left[\tilde{Q}_{i}\right]_{i=1}^{h}\end{array}\right]>0$, with $\bar{Q}_{i} \in \mathbb{R}^{n_{i} \times n_{i}}, \tilde{Q}_{i} \in \mathbb{R}^{m_{i} \times m_{i}}, \hat{Q}_{i} \in \mathbb{R}^{n_{i} \times m_{i}}$, $X_{1}, X_{2}$ and $X_{3}=\left[\begin{array}{c}\Gamma \circ X_{L} \\ \Gamma \circ X_{D}\end{array}\right]$, with $X_{L} \in \mathbb{R}^{n \times p}, X_{D} \in \mathbb{R}^{m \times p}$, and scalars $\varepsilon_{i j}>0, i=1, \cdots, h, j=1, \cdots, h$, and $\rho>0$ satisfying the following LMI:

$$
\left[\begin{array}{ccccccccc}
\check{\chi}_{11} & \star & \star & \star & \star & \star & \star & \cdots & \star \\
0 & \bar{\chi}_{22} & \star & \star & \star & \star & \star & \cdots & \star \\
\sqrt{2} H^{T} P\left(G-\Gamma \circ G_{\lambda}\right) & \sqrt{2} H^{T} P\left[\Gamma \circ G_{\lambda} H\right] & -H^{T} P H & \star & \star & \star & \star & \cdots & \star \\
Q[G-\Gamma \circ G] & Q G_{t}-X_{3} C_{t} & 0 & -Q & \star & \star & \star & \cdots & \star \\
P G+H X_{1} & 0 & 0 & 0 & -P & \star & \star & \cdots & \star \\
H X_{2} & 0 & 0 & 0 & 0 & -P & \star & \cdots & \star \\
0 & 0 & \sqrt{2} \Xi_{1}^{T} P H & {\left[\Xi_{1}^{T} 0\right] Q} & \Xi_{1}^{T} P & 0 & -\mathrm{Y}_{1} & \ldots & \star \\
\vdots & \vdots & \vdots & \vdots & \vdots & \vdots & \vdots & \ddots & \vdots \\
0 & 0 & \sqrt{2} \Xi_{h}^{T} P H & {\left[\Xi_{h}^{T} 0\right] Q} & \Xi_{h}^{T} P & 0 & 0 & \cdots & -\mathrm{Y}_{h}
\end{array}\right]
$$

where $0<P_{i_{11}} \in \mathbb{R}^{m_{i} \times m_{i}}, 0<P_{i_{22}} \in \mathbb{R}^{\left(n_{i}-m_{i}\right) \times\left(n_{i}-m_{i}\right)}$ and $U_{i} \in \mathbb{R}^{n_{i} \times n_{i}}$ is defined in Lemma 5 in [24], $\check{\chi}_{11}=-P+X_{2}^{T} H^{T}+$ $H X_{2}+\rho I+\sum_{i=1}^{h} \mathrm{Y}_{i} \Lambda_{i}^{T} \Lambda_{i}$, with $\mathrm{Y}_{i}=\operatorname{diag}\left[\varepsilon_{i j} I_{n_{j}}\right]_{j=1}^{h}, \bar{\chi}_{22}=-Q+\rho I, \Gamma=\left[\gamma_{i j}\right]_{h \times h}$ is a given structure matrix and $\{\star\}$ denotes the symmetric elements in a symmetric matrix. Here $S=H^{T} P$ and the observer gain is

$$
L_{t}=Q^{-1} X_{3}
$$

Proof. Define

$$
V(\zeta(k))=x^{T}(k) P x(k)+e_{t}^{T}(k) Q e_{t}(k)+\sigma^{T}(k)(S H)^{-1} \sigma(k),
$$

where $\zeta(k)=\left[x^{T}(k) e_{t}^{T}(k) \sigma^{T}(k)\right]^{T}, P>0$ and $Q>0$ are symmetric matrices and $S=H^{T} P$. Note that the inclusion of both state $x(k)$ and sliding function $\sigma(k)$ in the Lyapunov candidate function makes it possible to analyse simultaneously the reachabiltiy of QSM as well as the boundedness of the system state and sliding function, as will be seen later in the proof of Theorem 2. Defining

$$
\varpi=\left[\begin{array}{lll}
x^{T}(k) & e_{t}^{T}(k) & \bar{\xi}(k+1)
\end{array}\right]^{T},
$$

it can be written

$$
\begin{aligned}
\Delta V(\zeta(k)) & \triangleq V(\zeta(k+1))-V(\zeta(k)) \\
& =\varpi^{T}(k)\left[\chi_{i j}\right]_{3 \times 3} \varpi(k) .
\end{aligned}
$$

where

$$
\begin{aligned}
& \chi_{11}=(G+\Delta G)^{T} P(G+\Delta G)-(G+\Delta G)^{T} P H\left(H^{T} P H\right)^{-1} H^{T} P(G+\Delta G) \\
& -P H\left(H^{T} P H\right)^{-1} H^{T} P-P+2\left(\Delta G+G-\Gamma \circ G_{\lambda}\right)^{T} P H\left(H^{T} P H\right)^{-1} \\
& \times H^{T} P\left(\Delta G+G-\Gamma \circ G_{\lambda}\right)+\left[\begin{array}{c}
\Delta G+G-\Gamma \circ G_{\lambda} \\
0
\end{array}\right]^{T} Q\left[\begin{array}{c}
\Delta G+G-\Gamma \circ G_{\lambda} \\
0
\end{array}\right], \\
& \chi_{12}=2\left(\Delta G+G-\Gamma \circ G_{\lambda}\right)^{T} P H\left(H^{T} P H\right)^{-1} H^{T} P\left[\Gamma \circ G_{\lambda} \quad H\right] \\
& +\left[\begin{array}{c}
\Delta G+G-\Gamma \circ G_{\lambda} \\
0
\end{array}\right]^{T} Q\left(G_{t}-L_{t} C_{t}\right), \\
& \chi_{13}=\left[\begin{array}{c}
\Delta G+G-\Gamma \circ G_{\lambda} \\
0
\end{array}\right]^{T} Q \\
& \chi_{22}=2\left[\begin{array}{ll}
\Gamma \circ G_{\lambda} & H
\end{array}\right]^{T} S^{T}(S H)^{-1} S\left[\begin{array}{cc}
\Gamma \circ G_{\lambda} & B
\end{array}\right]+\left(G_{t}-L_{t} C_{t}\right)^{T} Q\left(G_{t}-L_{t} C_{t}\right)-Q, \\
& \chi_{23}=\left(G_{t}-L_{t} C_{t}\right)^{T} Q \text {, } \\
& \chi_{33}=Q \text {. }
\end{aligned}
$$

Now, in order to analyse the system stability let $\bar{\xi}(k+1)=0$. The system will be stable if

$$
\Xi:=\left[\chi_{i j}\right]_{2 \times 2}<-\rho I
$$


where $\rho>0$ is a scalar variable. To consider the feasibility of (23), by the Schur complement, (23) is equivalent to

$$
\left[\begin{array}{cccc}
\bar{\chi}_{11} & \star & \star & \star \\
0 & \bar{\chi}_{22} & \star & \star \\
\sqrt{2} H^{T} P(G+\Delta G-\Gamma \circ G) & \sqrt{2} H^{T} P\left[\Gamma \circ G_{\lambda} H\right] & -H^{T} P H & \star \\
Q[G+\Delta G-\Gamma \circ G] & Q\left(G_{t}-L_{t} C_{t}\right) & 0 & -Q
\end{array}\right]<0,
$$

where

$$
\begin{aligned}
\bar{\chi}_{11}= & (G+\Delta G)^{T} P(G+\Delta G)-(G+\Delta G)^{T} S^{T}(S H)^{-1} S(G+\Delta G) \\
& -S^{T}(S H)^{-1} S-P+\rho I, \\
\bar{\chi}_{22}= & -Q+\rho I .
\end{aligned}
$$

Consequently, Lemma 2.1 in [25] can be used to show that the feasibility of (24) is equivalent to that of

$$
\left[\begin{array}{cc}
\hat{\chi}_{11} & \cdots \\
\vdots & \ddots
\end{array}\right]<0
$$

with

$$
\begin{aligned}
\hat{\chi}_{11}= & \left(G+\Delta G+H F_{1}\right)^{T} P\left(G+\Delta G+H F_{1}\right)-P \\
& +F_{2}^{T}\left(H^{T} P H\right) F_{2}+F_{2}^{T} H^{T} P+P H F_{2}+\rho I,
\end{aligned}
$$

where $F_{1}$ and $F_{2}$ are auxiliary variables [26] and note that except $\hat{\chi}_{11}$, other elements of (25) are the same as those in (24). Therefore, using Lemma 5 in [24], $\hat{\chi}_{11}$ in (26) can be rearranged as

$$
\begin{aligned}
\hat{\chi}_{11}= & {\left[P(G+\Delta G)+H Z F_{1}\right]^{T} P^{-1}\left[P(G+\Delta G)+H Z F_{1}\right]-P } \\
& +F_{2}^{T} Z^{T} H^{T} P^{-1} H Z F_{2}+F_{2}^{T} Z^{T} H^{T}+H Z F_{2}+\rho I,
\end{aligned}
$$

where $Z$ satisfies $P H=H Z$. Defining $X_{1}=Z F_{1}, X_{2}=Z F_{2}$ and $X_{3}=Q L_{t}$, with the help of the Schur complement and Lemma 1 in [24], it can be seen that (25) is sufficed by the LMI in (19).

Remark 4. The LMI condition (19), used to obtain the controller parameters, is derived using a lossless technique in Lemma 2.1 of [25], leading to a less conservative stability condition compared to the current literature which uses trivial inequalities to make a convex platform for the controller synthesis. However, it is worth noting that the block diagonal structure of the symmetric positive definite matrix $P$, required to (i) form a decentralised sliding surface (i.e. $S=H^{T} P$ ), and (ii) satisfies $P H=H Z$, as well as the certain structure of the symmetric positive definite matrix variable $Q$, necessary to keep the structure of the state and disturbance observer gains same as the structure matrix $\Gamma$, can potentially introduce conservatism to the problem. Other non-convex or convex approaches may be proposed in future that is less conservative.

The following theorem aims to characterise the boundedness of the obtained overall closed-loop system state and corresponding decentralised sliding function in the presence of disturbance $\xi(k)$.

Theorem 2. In the presence of disturbance $\xi(k)$, if the LMI in (19) is feasible, for the obtained $P, Q, L_{t}=Q^{-1} X_{3}$ and $\rho$, the controller (14) satisfying (7) leads to a bound on the augmented system state $\zeta(k)=\left[x^{T}(k), e_{t}^{T}(k), \sigma^{T}(k)\right]^{T}$ as follows:

$$
\forall \varsigma>0, \exists k^{\star}>0, \text { s.t. } \forall k>k^{\star},\|\zeta(k)\|^{2} \leq \frac{\lambda_{\max }(\mathbf{M})}{\hat{\rho} \lambda_{1}} \delta+\varsigma,
$$

where $\lambda_{1}=\lambda_{\min }\left(\operatorname{diag}\left(P, Q,\left(H^{T} P H\right)^{-1}\right)\right), \mathbf{M}=\operatorname{diag}\left(M_{P}, Q\right), M_{P}=P H\left(H^{T} P H\right)^{-1} H^{T} P+P$, and $\delta=\|\Pi+Q\| \sum_{i=1}^{h} \tilde{L}_{i}^{2} T_{s}^{2}$; here the scalar variable $\hat{\rho}>0$ and matrix variable $\Pi>0$ are obtained from solving the following LMI:

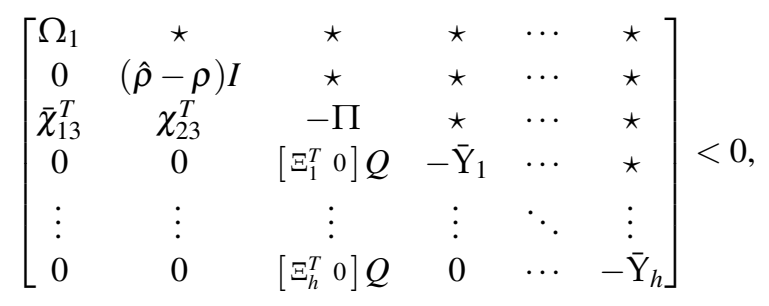

where $\bar{\chi}_{13}=\left[\begin{array}{c}G-\Gamma \circ G_{\lambda} \\ 0\end{array}\right]^{T} Q, \chi_{23}=\left(G_{t}-L_{t} C_{t}\right)^{T} Q, \Omega_{1}=(\hat{\rho}-\rho) I+\sum_{i=1}^{h} \bar{Y}_{i} \Lambda_{i}^{T} \Lambda_{i}$ and $\bar{Y}_{i}=\operatorname{diag}\left[\bar{\varepsilon}_{i j} I_{n_{j}}\right]_{j=1}^{h}$ in which $\bar{\varepsilon}_{i j}>0, i=1, \cdots, h, j=1, \cdots$, h are scalar variables. 
Proof. Defining

$$
\begin{aligned}
v(k) & =\left[\begin{array}{ll}
x^{T}(k) & e_{t}^{T}(k)
\end{array}\right]^{T}, \\
\chi_{v} & =\left[\begin{array}{ll}
\chi_{13}^{T} & \chi_{23}^{T}
\end{array}\right]^{T},
\end{aligned}
$$

and according to Lemma 4 in [27] it can be written that

$$
2 v^{T}(k) \chi_{v} \bar{\xi}(k+1) \leq v^{T}(k) \chi_{v} \Pi^{-1} \chi_{v}^{T} v(k)+\bar{\xi}^{T}(k+1) \Pi \bar{\xi}(k+1)
$$

where $\Pi>0$ is of appropriate dimension. It follows from (22), (23) and (30) that

$$
\Delta V(\zeta(k)) \leq-v^{T}(k)\left[\rho I-\chi_{v} \Pi^{-1} \chi_{v}^{T}\right] v(k)+\bar{\xi}^{T}(k+1)\left[\chi_{33}+\Pi\right] \bar{\xi}(k+1) .
$$

If we choose $\Pi>0$ such that

$$
\hat{\rho} I<\rho I-\chi_{v} \Pi^{-1} \chi_{v}^{T},
$$

where $\rho>\hat{\rho}>0$, which is always possible if $\rho>0$ exists, then, it follows from (31) that

$$
\Delta V(\zeta(k)) \leq-\hat{\rho} v^{T}(k) v(k)+\bar{\xi}^{T}(k+1)\left[\chi_{33}+\Pi\right] \bar{\xi}(k+1)
$$

Moreover, note that

$$
V(\zeta(k))=v^{T}(k) \mathbf{M} v(k),
$$

where $\mathbf{M}=\operatorname{diag}\left(M_{P}, Q\right)$, and $M_{P}=P H\left(H^{T} P H\right)^{-1} H^{T} P+P$, hence,

$$
\lambda_{\min }(\mathbf{M})\|v(k)\|^{2} \leq V(\zeta(k)) \leq \lambda_{\max }(\mathbf{M})\|v(k)\|^{2} .
$$

Additionally, it is known that

$$
\lambda_{1}\|\zeta(k)\|^{2} \leq V(\zeta(k)) \leq \lambda_{2}\|\zeta(k)\|^{2} .
$$

where $\lambda_{1}=\lambda_{\min }\left(\operatorname{diag}\left(P, Q,\left(H^{T} P H\right)^{-1}\right)\right)$ and $\lambda_{2}=\lambda_{\max }\left(\operatorname{diag}\left(P, Q,\left(H^{T} P H\right)^{-1}\right)\right)$. Hence, from (33) and (35) one can derive that

$$
\Delta V(\zeta(k)) \leq-\frac{\hat{\rho}}{\lambda_{\max }(\mathbf{M})} V(\zeta(k))+\delta,
$$

where $\delta=\|\Pi+Q\| \sum_{i=1}^{h} \tilde{L}_{i}^{2} T_{s}^{2}$. Moreover, from (23) it can simply be written that $\forall v(k) \neq 0$

$$
\begin{aligned}
v^{T}(k) \Xi v(k) & =\left.V(\zeta(k+1))\right|_{\bar{\xi}_{(k+1)=0}}-V(\zeta(k)) \\
& <-\rho v^{T}(k) v(k) .
\end{aligned}
$$

It is known that $\left.V(\zeta(k+1))\right|_{\bar{\xi}_{(k+1)=0}} \geq 0$, and thus, from (38) and (35), it can be claimed that $\rho<\lambda_{\max }(\mathbf{M})$. Therefore, $\frac{\hat{\rho}}{\lambda_{\max }(\mathbf{M})}<1$. Finally, from [28] (Theorem 5.1, Corollaries 5.1, 5.2) and (37), one can find the bound given in (28). Moreover, to find $\Pi>0$ in (32), for given $P>0, Q>0, L_{t}=Q^{-1} X_{3}$ and $\rho>0$, by using Lemma 1 in [24], we can show that (32) is sufficed by the LMI in (29).

As seen in the proposed sparse distributed ODSMC, local controllers/observers are able to utilise some interconnections in the nominal $G$ matrix, and the remaining interconnections in $G$ matrix together with $\Delta G$ are considered as the uncertainties of the overall system. The second step of this paper will consider the issue of minimising the costs of a control/observer network utilised for the stabilising distributed ODSMC. This will be the subject of the next section.

Remark 5. It is easy to realise from Lemma 1 and 2 that $\mathrm{S}\left(Q^{-1}\right)=\mathrm{S}(Q)$, and thus $\mathrm{S}\left(L_{S}\right) \subseteq \Gamma, \mathrm{S}\left(D_{S}\right) \subseteq \Gamma$.

\section{Identifying The Most Sparse Stabilising Control Network}

The previous section has developed an LMI based framework for the design of distributed ODSMC for NCSs while assuming a priori known structural constraint on communication requirements between sub-systems. This section aims to design a control network with the minimum number of links that satisfies the stability condition (19). Note that the main reason that we consider network sparsification problem here is the minimisation of the cost of the control network utilised to stabilise the system. As it is assumed that the general costs, including the construction and data transferring costs etc, are identical for all the links, minimising the costs of a control network can intuitively be considered as minimising the number 
of communication links in the control network structure or equivalently finding the sparsest control network structure that can stabilise the system. We formulate this problem as

$$
\begin{aligned}
& \min _{P, Q, X_{1}, X_{2}, X_{3}, \rho, \mathrm{Y}_{1}, \cdots, \mathrm{Y}_{h}} \operatorname{card}(\Gamma) \\
& \text { subject to (19) and } \Gamma \subseteq \mathrm{S}(G),
\end{aligned}
$$

where $\Gamma=\left[\gamma_{i j}\right]_{h \times h}$ and $\operatorname{card}(\cdot)$ denotes the cardinality function (the number of nonzero elements of a matrix). The above optimisation problem is a convex mixed-binary problem which is broadly speaking NP-hard. A number of exact schemes for addressing the convex mixed-binary programs are considered [29]. However, exact schemes require an exhaustive search whose computation time grows faster than polynomial, as the number of communication links increases. In our case, in the worst case, one should solve $2^{\mathrm{N}}$ convex problems, where $\mathrm{N}$ denotes the number of physical interconnections in the plant network, to achieve the exact solution. Instead, in this paper, a heuristic sub-optimal scheme is proposed that is able to successively deal with this problem.

As explained in [10], to avoid performing an exhaustive search, a trade-off can be made either in the choice of the search strategy or in the choice of the selection criterion. Another alternative to avoid solving a combinatorial problem is to consider a multi-objective problem of controller structure and control law co-design by incorporating secondary cost functions which are convex approximations of the original $\ell_{0}$-quasi-norms and can promote sparsity of the distributed controller, into a main cost function, which represents a performance specification of the closed-loop system [7, 11]. The reweighted $\ell_{1}$ (REL1) norm algorithms can be further employed to identify the sparse optimal feedback gain [7]. The weights (entries of the weighting matrix), in the REL1 algorithms are updated at each iteration based on the solutions of the previous iteration. It should be emphasised that the existing reweighted algorithms cannot be employed to address the optimisation problem in (39), as they cannot handle binary matrix variable.

In this paper, in order to identify a control network with the minimum number of links that satisfies the stability condition (19), we propose an algorithm presented below. Note that in this algorithm, we relax the constraint on $\gamma_{i j}, i \neq j$ from binary variables to the constraint $0 \leq \gamma_{i j} \leq 1$.

\section{Algorithm 1.}

1) Set $\Gamma=I$. If the LMI feasibility problem in (19) is feasible with respect to $P>0, Q>0, X_{1}, X_{2}, X_{3}, \rho>0, Y_{1}>$ $0, \cdots, \mathrm{Y}_{h}>0,\left\{\gamma_{i j}^{\star}\right\} \leftarrow\left\{\gamma_{i j}\right\}$, no control network is required and the sparsest structure is the decentralised structure. Terminate the search and go to Step 6.

2) Initialise $\Gamma=\mathrm{S}(G)$ and $\mathbf{s}=1$, in which $\mathbf{s}$ denotes the iteration number.

3) Solve the LMI feasibility problem in (19) to find $P>0, Q>0, X_{1}, X_{2}, X_{3}, \rho>0, \mathrm{Y}_{1}>0, \cdots, \mathrm{Y}_{h}>0$. If it is feasible, $\left\{\gamma_{i j}^{\star}\right\} \leftarrow\left\{\gamma_{i j}\right\}$. Otherwise, if $\mathbf{s}=1$ terminate the search and the problem has no solution, or else go to Step 6.

4) With known $P, Q, X_{1}, X_{2}, X_{3}, \rho, \mathrm{Y}_{1}, \cdots, \mathrm{Y}_{h}$ and replacing those entries $\gamma_{i j}=1, i \neq j$ with the relaxed constraints $0 \leq \gamma_{i j} \leq 1$, minimise $\sum_{i, j=1, i \neq j}^{h} \gamma_{i j}$ subject to the LMI (19) and $0 \leq \gamma_{i j} \leq 1$ to find the relaxed $\gamma_{i j}^{r}$. Sort the set $\left\{\gamma_{i j}^{r}\right\}$ in ascending order.

5) Set $\gamma_{i j}$ corresponding to the first entry of $\left\{\gamma_{i j}^{r}\right\}$ to zero and $\mathbf{s}=\mathbf{s}+1$. If $\mathbf{s}<\mathbf{C a r d}(\mathrm{S}(G))-n$, return to Step 3, otherwise go to Step 6.

6) Return $\gamma_{i j}^{\star}$.

In the above algorithm, Step 4 characterises the contribution of each link in the stability of the overall system. Moreover, as seen, the algorithm searches for the sparsest structure using the sorted set $\left\{\gamma_{i j}^{r}\right\}$. In the worst case, in order to find the solution, $2(\operatorname{Card}(\mathrm{S}(G))-n)$ convex problems may be addressed. Finally, it should be stressed that this alternate scheme is only a sub-optimal method to deal with the sparsification problem considered in this section. Broadly speaking, to obtain the optimal solution, one should solve the original mixed-binary convex problem in (39), which is NP-hard.

\section{NUMERICAL EXAMPLES}

In order to evaluate the proposed theory, three numerical examples are given in this section. In order to evaluate the proposed theory, two numerical examples are given in this section. In the first example, which is from the reference [4], the underlying interconnected system consists of three inverted pendulums that are mounted on coupled carts. It is shown that the control network topolgy with the minimum number of communication links that can stabilise this system is the decentralised structure, i.e. the local controllers does not need to receive other subsystems' information. We consider a second example to analyse the cases that the decentralised structure is not stabilising, and thereby evaluate the performance of Algorithm 1 proposed to sparsify the control network. We used YALMIP [30] as the interface in Matlab, as well as SDPT3 [31] as the LMI solver to solve the LMI feasibility problems. 


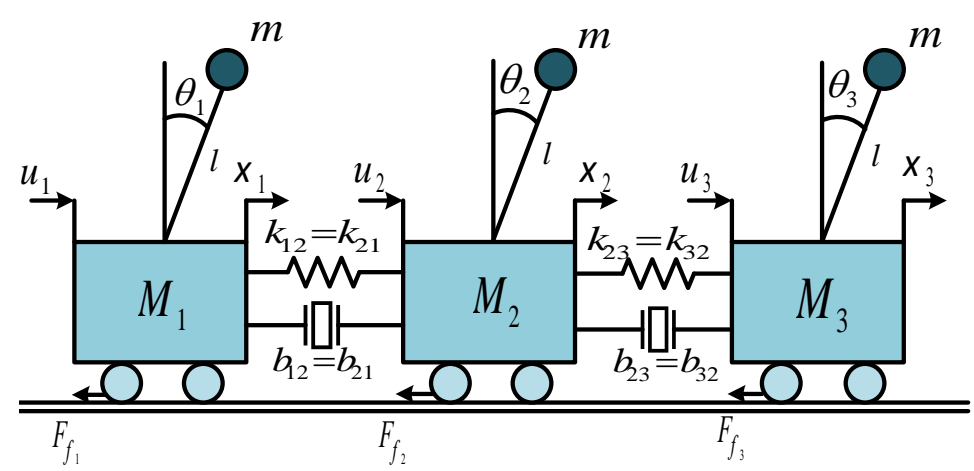

Figure 1: Three coupled inverted pendulums system

\section{i. Example 1}

Consider an interconnected system consisting of three inverted pendulums that are mounted on coupled carts [4, 32], shown in Fig. 1. The linearised equations of motions are [4]:

$$
\begin{aligned}
& M_{i} l \ddot{\theta}_{i}=\left(M_{i}+m\right) g \theta_{i}+c_{i} \dot{\mathbf{x}}_{i}+\sum_{j=1, j \neq i}^{h}\left[b_{i j}\left(\dot{\mathbf{x}}_{i}-\dot{\mathbf{x}}_{j}\right)+k_{i j}\left(\mathbf{x}_{i}-\mathbf{x}_{j}\right)\right]-u_{i}, \\
& M_{i} \ddot{\mathbf{x}}_{i}=-c_{i} \dot{\mathbf{x}}_{i}-\sum_{j=1, j \neq i}^{h}\left[b_{i j}\left(\dot{\mathbf{x}}_{i}-\dot{\mathbf{x}}_{j}\right)+k_{i j}\left(\mathbf{x}_{i}-\mathbf{x}_{j}\right)\right]-m g \theta_{i}+u_{i},
\end{aligned}
$$

where $k_{i j}=k_{j i}, b_{i j}=b_{j i}, c_{i}$ and $l$ are spring, damper, friction coefficients, and pendulum length, respectively. Here, it is assumed that the moment of inertia of the pendulums is zero. Define $x_{i}=\left[x_{i, 1}, x_{i, 2}, x_{i, 3}, x_{i, 4}\right]^{T}=\left[\theta_{i}, \dot{\theta}_{i}, \mathbf{x}_{i}, \dot{\mathbf{x}}_{i}\right]^{T}$, and now the system in (40) is rearranged as an interconnected state space in continuous-time with the system matrices as below:

$$
\begin{aligned}
A_{i i} & =\left[\begin{array}{cccc}
0 & 1 & 0 & 0 \\
\frac{M_{i}+m}{M_{i} l} g & 0 & \frac{k_{i}}{M_{i} l} & \frac{c_{i}+b_{i}}{M_{i} l} \\
0 & 0 & 0 & 1 \\
\frac{-m}{M_{i}} g & 0 & \frac{-k_{i}}{M_{i}} & \frac{-c_{i}-b_{i}}{M_{i} l}
\end{array}\right], A_{i j}=\left[\begin{array}{cccc}
0 & 0 & 0 & 0 \\
0 & 0 & \frac{-k_{i j}}{M_{i} l} & \frac{-b_{i j}}{M_{i} l} \\
0 & 0 & 0 & 0 \\
0 & 0 & \frac{k_{i j}}{M_{i}} & \frac{b_{i j}}{M_{i}}
\end{array}\right] \\
B_{i} & =\left[\begin{array}{llll}
0 & \frac{-1}{M_{i} l} & 0 & \frac{1}{M_{i}}
\end{array}\right]^{T}, C_{i}=\left[\begin{array}{cccc}
1 & 0 & 0 & 0 \\
0 & 0 & 1 & 0
\end{array}\right],
\end{aligned}
$$

for $(i, j) \in \mathbf{E} \triangleq\{(1,2),(2,1),(2,3),(3,2)\}$. Here $k_{i}=\sum_{j \in J_{i}} k_{i j}$ and $b_{i}=\sum_{j \in J_{i}} b_{i j}$, where $J_{i}:=\{j \mid(i, j) \in \mathbf{E}\}$. The numerical system parameters are assumed as $M_{1}=2, M_{2}=1, M_{3}=3, m=0.5, g=10, l=0.5, k_{12}=k_{21}=5, k_{23}=$ $k_{32}=15, b_{12}=b_{21}=1, b_{23}=b_{32}=5, c_{1}=4, c_{2}=2$ and $c_{3}=1$. A discretised representation based on a sample interval of $0.005 s$ is obtained. We set $\lambda_{i}=0.7$. In order to check the robustness properties of the controller, the following uncertainty parameters are considered: $\Xi_{i j}=0.01 \times \mathbf{1}_{4 \times 1}, \Lambda_{i j}=-0.01 \times \mathbf{1}_{1 \times 4}, i, j=1,2,3$. Algorithm 1 is solved and then it is found that the most sparse structure that can satisfy the rank condition in Assumption 2 and, more importantly, the stability condition in the LMI (19) is the decentralised structure. For comparison, we then exploit an exhaustive search on the binary variables, followed by convex optimisation of other variables, and the obtained structure is also the decentralised one. We can see that the proposed sub-optimal algorithm leads to the same result as the optimal solution. 


\section{ii. Example 2}

Consider the system (1) with the following parameters:

$$
\begin{aligned}
& G=\left[\begin{array}{ccc|cc|cc}
0 & 0.2 & 0 & 0.2 & -0.1 & 0.2 & 0 \\
-0.3 & 1.45 & 0.3 & -0.1 & 0 & 0.03 & 0 \\
0.3 & 0 & 0.4 & -0.2 & 0.2 & -0.1 & 0 \\
\hline-0.03 & 0.1 & 0.1 & 0.05 & 0.2 & 0 & 0.2 \\
0 & 0.1 & 0.05 & 0 & 1.3 & 0.1 & -0.1 \\
\hline 0 & 0.2 & 0.1 & 0 & 0 & 0.1 & -0.3 \\
0 & -0.2 & 0.2 & -0.2 & 0 & 0 & 1.1
\end{array}\right], \\
& H=\left[\begin{array}{cc|c|c}
1 & 0 & 0 & 0 \\
0 & 0.5 & 0 & 0 \\
2 & 0 & 0 & 0 \\
\hline 0 & 0 & 1 & 0 \\
0 & 0 & 1 & 0 \\
\hline 0 & 0 & 0 & 1 \\
0 & 0 & 0 & 2
\end{array}\right], C=\left[\begin{array}{ccc|cc|cc}
-10 & 2 & 1 & 0 & 0 & 0 & 0 \\
1 & -3 & -1 & 0 & 0 & 0 & 0 \\
\hline 0 & 0 & 0 & 1 & 0 & 0 & 0 \\
0 & 0 & 0 & -2 & 1 & 0 & 0 \\
\hline 0 & 0 & 0 & 0 & 0 & 2 & 1
\end{array}\right], \\
& \Xi_{11}=\left[\begin{array}{c}
0.1 \\
0.02 \\
0.1
\end{array}\right], \Xi_{12}=\left[\begin{array}{c}
0.1 \\
-0.1 \\
0
\end{array}\right], \Xi_{13}=\left[\begin{array}{c}
0.01 \\
0 \\
-0.01
\end{array}\right], \quad \Xi_{21}=\left[\begin{array}{l}
0.1 \\
0.2
\end{array}\right], \Xi_{22}=\left[\begin{array}{c}
0 \\
-0.2
\end{array}\right] \text {, } \\
& \Xi_{23}=\left[\begin{array}{c}
-0.01 \\
0
\end{array}\right], \Xi_{31}=\left[\begin{array}{c}
0.2 \\
-0.01
\end{array}\right], \Xi_{32}=\left[\begin{array}{c}
-0.01 \\
0
\end{array}\right], \Xi_{33}=\left[\begin{array}{l}
0.1 \\
0.2
\end{array}\right] \text {, } \\
& \Lambda_{11}^{T}=\left[\begin{array}{c}
0.1 \\
0.1 \\
-0.1
\end{array}\right], \Lambda_{21}^{T}=\left[\begin{array}{c}
0.08 \\
0.1 \\
0.1
\end{array}\right], \Lambda_{31}^{T}=\left[\begin{array}{c}
0.01 \\
-0.02 \\
0
\end{array}\right], \Lambda_{12}^{T}=\left[\begin{array}{c}
0.05 \\
0
\end{array}\right], \Lambda_{22}^{T}=\left[\begin{array}{c}
0.05 \\
0.2
\end{array}\right] \\
& \Lambda_{32}^{T}=\left[\begin{array}{c}
0 \\
0.03
\end{array}\right], \Lambda_{13}^{T}=\left[\begin{array}{c}
0.01 \\
0.2
\end{array}\right], \Lambda_{23}^{T}=\left[\begin{array}{c}
-0.1 \\
0.2
\end{array}\right], \Lambda_{33}^{T}=\left[\begin{array}{c}
-0.02 \\
0
\end{array}\right] \text {, } \\
& \Theta_{i j}(k)=0.5 \sin (k), \lambda=0.8 .
\end{aligned}
$$

All three open-loop subsystems are unstable. Suppose

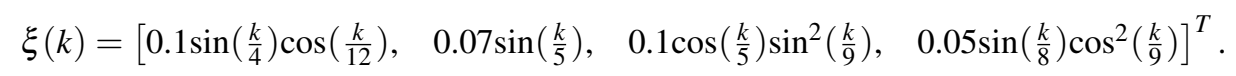

Solving the LMI feasibility problem in (19), by assuming a fully distributed structure for the control network; i.e. $\Gamma=\mathrm{S}(G)$, we obtain

$$
\begin{aligned}
& S=\left[\begin{array}{ccc|cc|cc}
0.0503 & -0.0331 & 0.1005 & 0 & 0 & 0 & 0 \\
-0.0033 & 0.0276 & -0.0066 & 0 & 0 & 0 & 0 \\
\hline 0 & 0 & 0 & 0.0466 & 0.0466 & 0 & 0 \\
\hline 0 & 0 & 0 & 0 & 0 & 0.0768 & 0.0829
\end{array}\right], \\
& L=\left[\begin{array}{cc|cc|c}
-0.0991 & -0.1359 & 0.0832 & -0.0411 & 0.0466 \\
-0.1752 & -0.7749 & -0.1106 & 0.0314 & 0.0526 \\
-0.2725 & -0.2341 & 0.3745 & 0.3325 & -0.0075 \\
\hline-0.0331 & -0.0892 & 1.3070 & 0.5230 & 0.1154 \\
-0.0342 & -0.1026 & 3.8072 & 1.8234 & -0.0135 \\
\hline-0.0496 & -0.1109 & -0.1630 & -0.0545 & 0.0914 \\
-0.0312 & 0.0566 & -0.5126 & -0.1075 & 0.9784
\end{array}\right] \\
& D=\left[\begin{array}{cc|cc|c}
-0.0759 & -0.0484 & 0.0826 & 0.0537 & -0.0040 \\
0.0009 & -0.1370 & -0.0544 & -0.0256 & -0.0021 \\
\hline-0.0060 & -0.0172 & 0.7952 & 0.2834 & 0.0039 \\
\hline-0.0083 & 0.0043 & -0.1712 & -0.0637 & 0.2050
\end{array}\right] \\
& \mathrm{Y}_{1}=\operatorname{diag}(0.3183,0.9880,0.0793) \text {, } \\
& \mathrm{Y}_{2}=\operatorname{diag}(0.2577,0.0889,0.0813) \text {, } \\
& \mathrm{Y}_{3}=\operatorname{diag}(1.6401,0.5180,1.1030) \text {, } \\
& \rho=4.4631 \times 10^{-4} \text {. }
\end{aligned}
$$



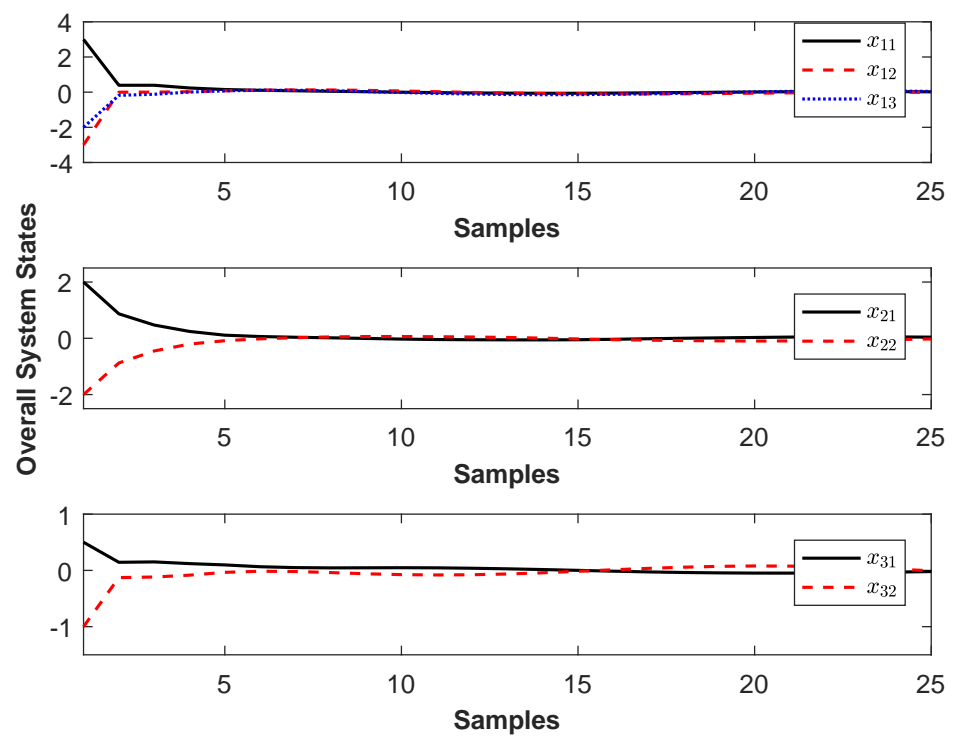

Figure 2: Trajectories of the system state with fully distributed structure

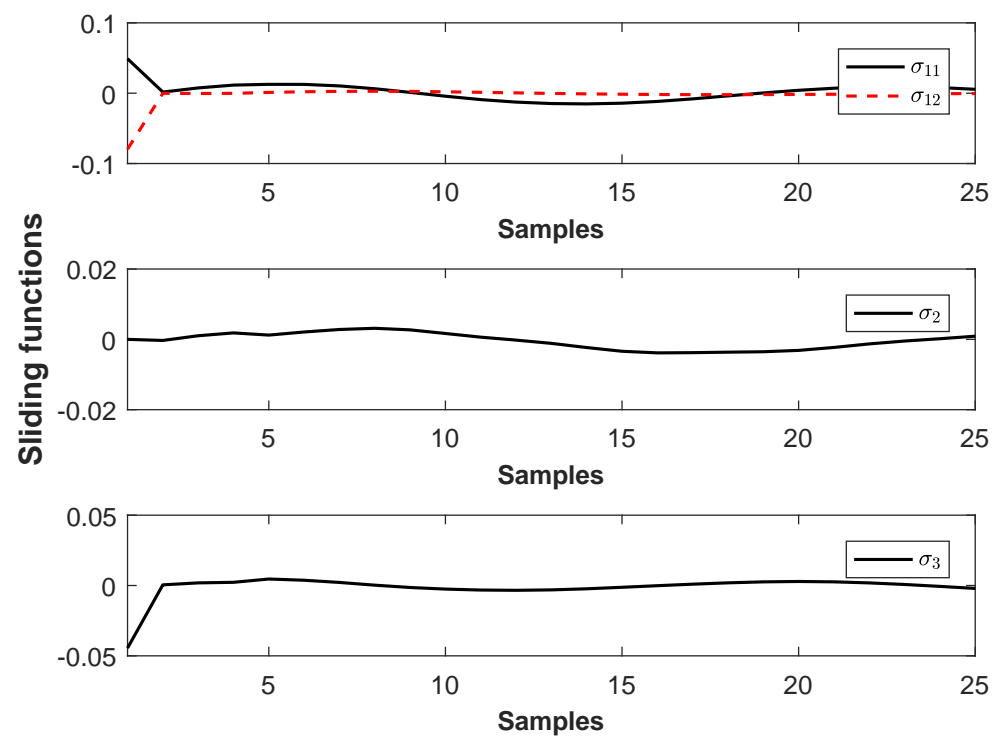

Figure 3: Deviation from the sliding surface with fully distributed structure

The control law and observer in (14) and (9) are derived by the achieved $S, L, D, \Gamma^{\star}$, and with $\lambda=0.8$. Applying this controller to the system (1), we illustrate the results in Figs. 2 - 4. Here, the initial state is assumed to be $x(0)=\left[\begin{array}{lllllll}3 & -3 & -2 & 2 & -2 & 0.5 & -1\end{array}\right]^{T}$. Fig. 5 shows the performance of the designed disturbance estimator. Now, we consider the problem of identifying the most sparse stabilising control network structure. Firstly, the LMI in (19) is not feasible with the decentralised structure. Following the procedure given in Algorithm 1, the sparse structure matrix is obtained as

$$
\Gamma^{\star}=\left[\gamma_{i j}^{\star}\right]_{3 \times 3}=\left[\begin{array}{lll}
1 & 1 & 0 \\
0 & 1 & 0 \\
1 & 1 & 1
\end{array}\right] .
$$

It is worth mentioning that using an exhaustive search instead of the proposed simplified Algorithm 1 would result in the same structure. This demonstrates the effectiveness of the proposed sub-optimal algorithm in the paper for identifying a favourable sparse stabilising topology for the control network. Solving the LMI feasibility problem in (19), with the 

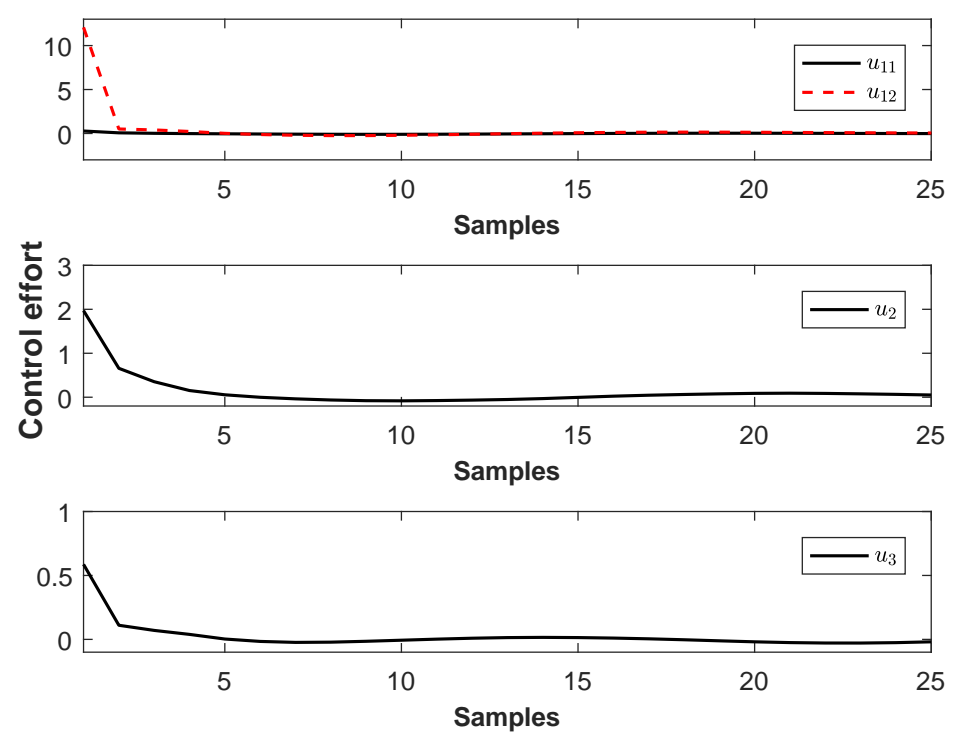

Figure 4: Control efforts with fully distributed structure

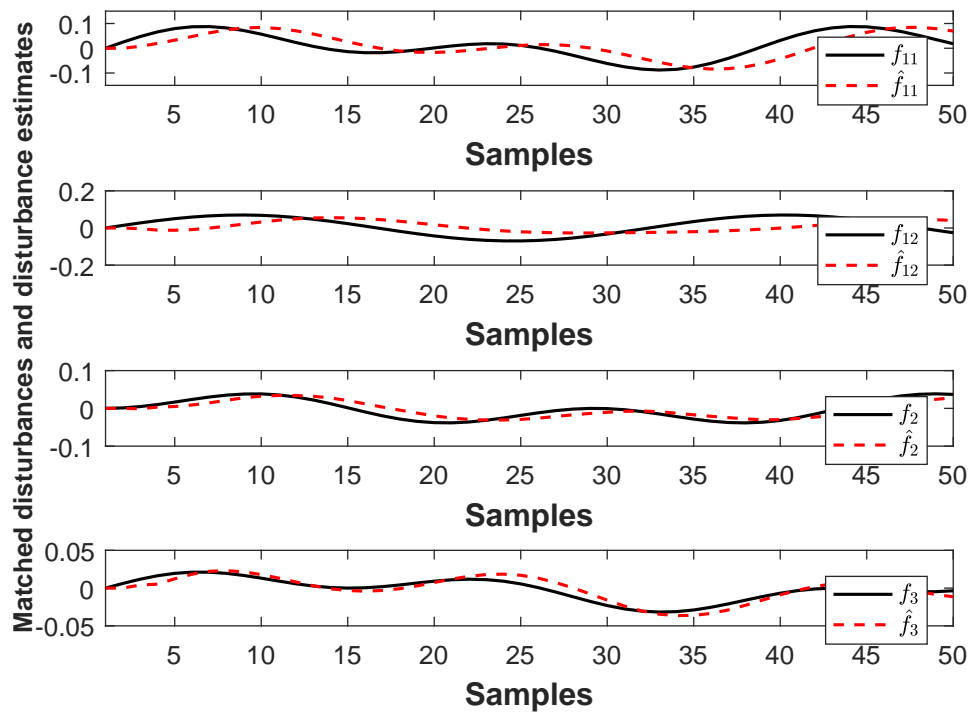

Figure 5: Exogenous disturbances and disturbance estimator outputs with fully distributed structure 


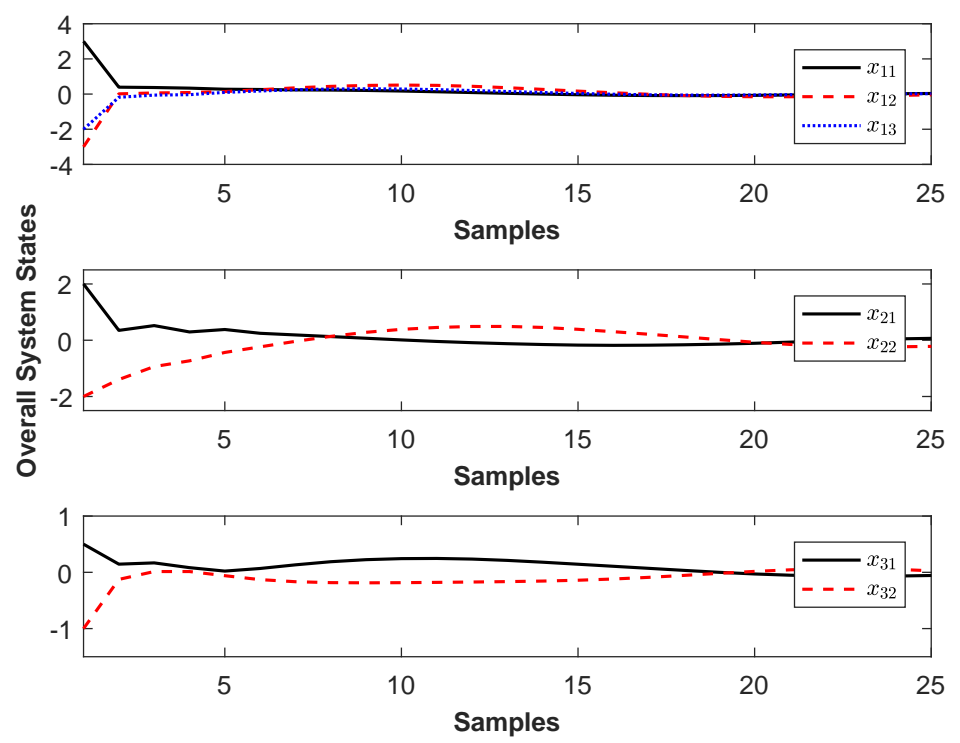

Figure 6: Trajectories of the system state with structure $\Gamma^{\star}$

structure constraint obtained previously $\left(\Gamma^{\star}\right)$, gives the parameters below:

$$
\begin{aligned}
& S=10^{-4} \times {\left[\begin{array}{ccc|cc|cc}
0.0340 & 0.0143 & 0.0679 & 0 & 0 & 0 & 0 \\
0.0014 & 0.0884 & 0.0029 & 0 & 0 & 0 & 0 \\
\hline 0 & 0 & 0 & 0.0220 & 0.0220 & 0 & 0 \\
\hline 0 & 0 & 0 & 0 & 0 & 0.6066 & 0.6702
\end{array}\right], } \\
& L= {\left[\begin{array}{cc|cc|c}
0.0080 & -0.0891 & -0.0994 & -0.0831 & 0 \\
0.0227 & -0.5051 & -0.1275 & 0.0042 & 0 \\
-0.0149 & -0.0253 & 0.2479 & 0.1975 & 0 \\
\hline 0 & 0 & 0.5244 & 0.4810 & 0 \\
0 & 0 & 3.0021 & 1.7892 & 0 \\
\hline-0.0069 & -0.0994 & 0.1756 & 0.0148 & -0.2955 \\
0.0408 & 0.1892 & -0.3746 & -0.0298 & 0.4391
\end{array}\right], } \\
& D= {\left[\begin{array}{cc|cc|c}
-0.0000 & -0.0020 & -0.0001 & 0.0000 & 0 \\
0.0000 & -0.0006 & 0.0000 & 0.0000 & 0 \\
\hline 0 & 0 & 0.0001 & 0.0001 & 0.00 \\
\hline 0.0000 & 0.0001 & -0.0002 & -0.0000 & 0.0002
\end{array}\right], } \\
& Y_{1}=10^{-3} \times \operatorname{diag}(0.0390,0.1996,0.0079), \\
& Y_{2}=10^{-5} \times \operatorname{diag}(0.6881,0.3360,0.0392), \\
& Y_{3}=\operatorname{diag}(0.0019,0.0000,0.0003),
\end{aligned}
$$

Using the given $S, L, D, \Gamma^{\star}$ and $\lambda=0.8$, the control law and observer given in (14) and (9), respectively, can be obtained. Figs. 6 - 8 demonstrate the closed-loop system state trajectories, deviation from sliding surface as well as control efforts, obtained by applying the controller to the system (1). As seen from these figures, the proposed sparse ODSMC law can successfully steer the state trajectories into a boundary layer about the ideal sliding surface and keep them there thereafter. However, it should also be noted that with more sparse structures, the ultimate bounds on the overall system state trajectories are wider compared to the less sparse structures. To evaluate this issue in a more quantitative way, we define

$$
E_{r m s} \triangleq \max _{i}\left(e_{r m s, i}\right), \quad i=1, \cdots, m,
$$

where $e_{r m s, i}$ is the discrete-time cumulative root mean square (RMS) value of $e_{\xi, i}$, as

$$
e_{r m s, i} \triangleq \sqrt{\frac{1}{N} \sum_{k=1}^{N}\left|e_{\xi, i}(k)\right|^{2}}, i=1, \cdots, m,
$$



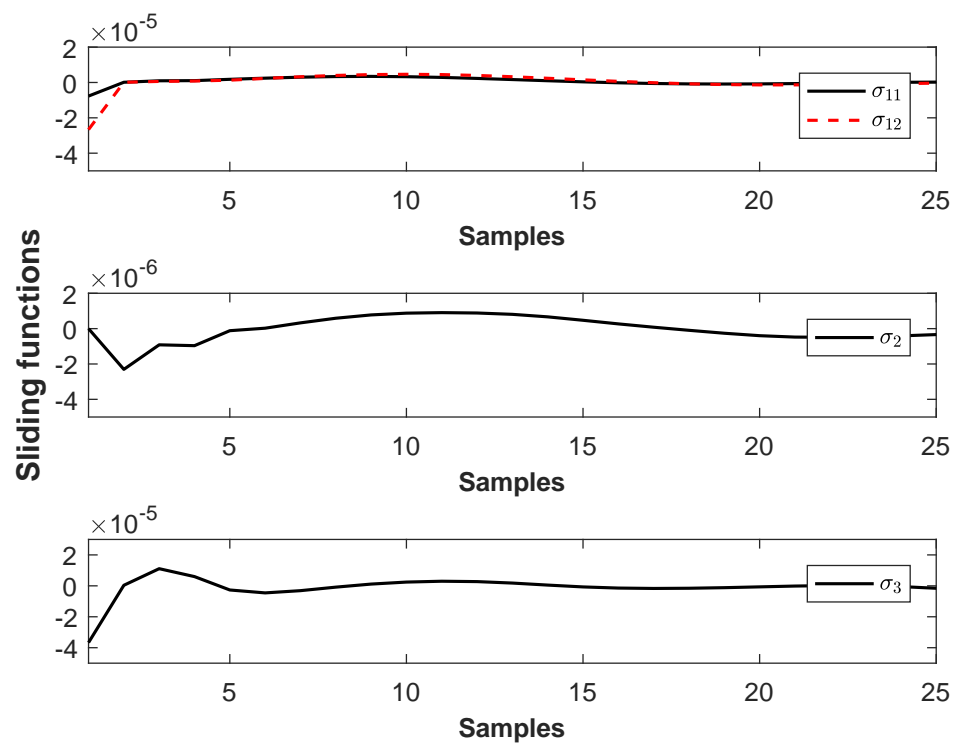

Figure 7: Deviation from the sliding surface with structure $\Gamma^{\star}$
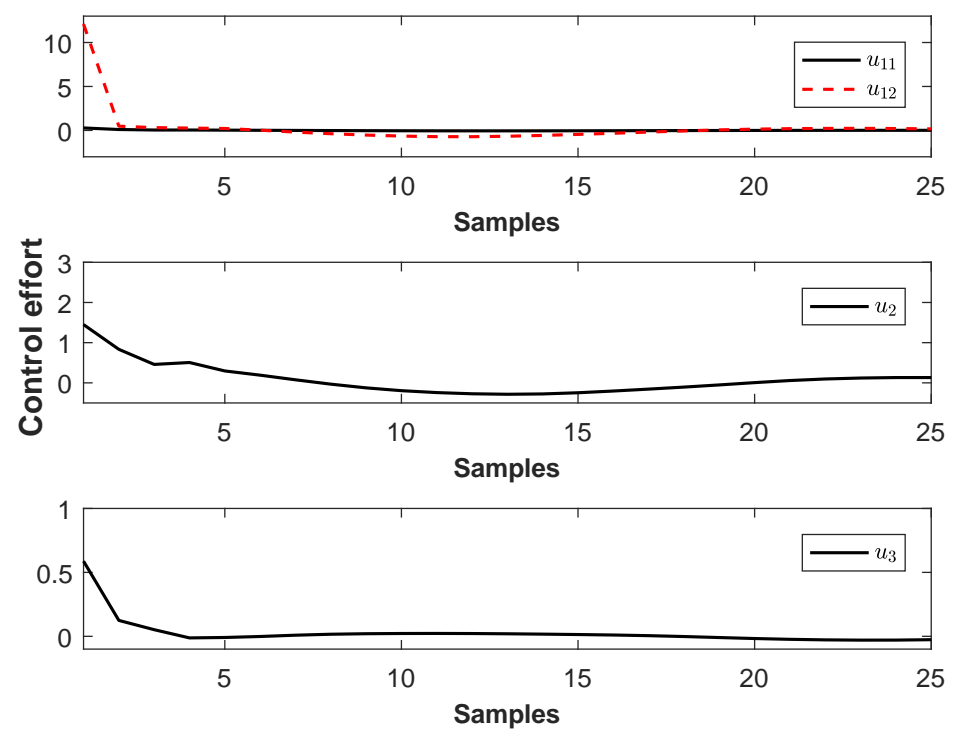

Figure 8: Control efforts with structure $\Gamma^{\star}$ 
where $N$ denotes the number of samples and $e_{\xi}$ is defined in (16). Table 1 presents $E_{r m s}$ corresponding to different structures. As seen from these results, the less sparse the control network structure, the narrower the ultimate bound on the state trajectories. This can also lead us to this conclusion that a trade off between the control performance and sparsity of

Table 1: Comparison of $E_{r m s}$ for different structures

\begin{tabular}{|c|c|c|c|c|}
\hline$\Gamma$ & {$\left[\begin{array}{lll}1 & 1 & 1 \\
1 & 1 & 1 \\
1 & 1 & 1\end{array}\right]$} & {$\left[\begin{array}{lll}1 & 1 & 1 \\
1 & 1 & 0 \\
1 & 1 & 1\end{array}\right]$} & {$\left[\begin{array}{lll}1 & 1 & 0 \\
1 & 1 & 0 \\
1 & 1 & 1\end{array}\right]$} & {$\left[\begin{array}{lll}1 & 1 & 0 \\
0 & 1 & 0 \\
1 & 1 & 1\end{array}\right]$} \\
\hline$E_{r m s}$ & 0.0066 & 0.0068 & 0.0076 & 0.0076 \\
\hline
\end{tabular}

the control network should be considered.

\section{CONCLUSIONS}

This paper firstly, with assuming a priori known structure for the control/observer network, has proposed an output feedback discrete time SMC for uncertain networked systems. A unified framework is derived for the observer-based controller design, with the aid of an LMI scheme, which has the ability to cover all the cases such as fully decentralised, fully distributed, and sparsely distributed topologies. Furthermore, our sparse ODSMC reduces the conservatism of the existing methods in the literature for the LMI based DSMC. Then, this paper has explored the solution to the problem of finding the sparsest control/observer network structure that satisfies the LMI stability condition obtained in the first part. The effectiveness of the proposed schemes has been evaluated by three numerical examples presented in the simulation section.

\section{REFERENCES}

[1] Zecevic A, Siljak D. Global low-rank enhancement of decentralized control for large-scale systems. IEEE Transactions on Automatic Control. 2005;50(5):740-744.

[2] Siljak D. Decentralized control of complex systems. Dover Publications; 2012.

[3] Wang X, Lemmon M. Event-triggering in distributed networked control systems. IEEE Transactions on Automatic Control. 2011;56(3):586-601.

[4] Razeghi-Jahromi M, Seyedi A. Stabilization of networked control systems with sparse observer-controller networks. IEEE Transactions on Automatic Control. 2015;60(6):1686-1691.

[5] Staroswiecki M, Amani AM. Fault-tolerant control of distributed systems by information pattern reconfiguration. International Journal of Adaptive Control and Signal Processing. 2014;

[6] Arastoo R, GhaedSharaf Y, Kothare MV, Motee N. Optimal state feedback controllers with strict row sparsity constraints. In: American Control Conference (ACC), 2016. IEEE; 2016. p. 1948-1953.

[7] Lin F, Fardad M, Jovanovic M. Augmented Lagrangian approach to design of structured optimal state feedback gains. IEEE Trans Autom Control. 2011;56(12):2923-2929.

[8] Zoltowski DM, Dhingra N, Lin F, Jovanovic MR. Sparsity-promoting optimal control of spatially-invariant systems. In: American Control Conference (ACC), 2014. IEEE; 2014. p. 1255-1260.

[9] Fardad M, Lin F, Jovanovic MR. Sparsity-promoting optimal control for a class of distributed systems. In: Proc. the American Control Conference. San Francisco, CA, USA; 2011. p. 2050-2055.

[10] Van De Wal M, De Jager B. A review of methods for input/output selection. Automatica. 2001;37(4):487-510.

[11] Schuler S, Münz U, Allgöwer F. Decentralized state feedback control for interconnected systems with application to power systems. Journal of Process Control. 2014;24(2):379-388.

[12] Yan XG, Edwards C, Spurgeon SK. Decentralized robust sliding mode control for a class of nonlinear interconnected systems by static output feedback. Automatica. 2004;40(4):613âĂŞ-620.

[13] Yan XG, Spurgeon SK, Edwards C. Decentralised sliding mode control for nonminimum phase interconnected systems based on a reduced-order compensator. Automatica. 2006;42(10):1821-1828.

[14] Qureshi A, Abido MA. Decentralized discrete-time quasi-sliding mode control of uncertain linear interconnected systems. International Journal of Control, Automation and Systems. 2014;12(2):349-357. 
[15] Mahmoud MS, Qureshi A. Decentralized sliding-mode output-feedback control of interconnected discrete-delay systems. Automatica. 2012;48(5):808-814.

[16] Amin S. Smart grid: Overview, issues and opportunities. advances and challenges in sensing, modeling, simulation, optimization and control. Euro Jour of Cont. 2011;17(5-6):547âĂŞ-567.

[17] Li S, Yang J, Chen Wh, Chen X. Disturbance observer-based control: methods and applications. CRC press; 2014.

[18] Su W, Drakunov SV, Özgüner Ü. An $\mathrm{O}\left(\mathrm{T}^{2}\right)$ boundary layer in sliding mode for sampled-data systems. IEEE Transactions on Automatic Control. 2000;45(3):482-485.

[19] Chang JL. Applying discrete-time proportional integral observers for state and disturbance estimations. Automatic Control, IEEE Transactions on. 2006;51(5):814-818.

[20] Milanese M, Vicino A. Optimal estimation theory for dynamic systems with set membership uncertainty: An overview. Automatica. 1991;27(6):997-1009.

[21] Lai NO, Edwards C, Spurgeon SK. Discrete output feedback sliding-mode control with integral action. Int J Robust Nonlinear Control. 2006;16:21-43.

[22] Edwards C. A practical method for the design of sliding mode controllers using linear matrix inequalities. Automatica. 2004;40:1761-1769.

[23] Argha A, Li L, Su SW, Nguyen H. On LMI-based sliding mode control for uncertain discrete-time systems. Journal of the Franklin Institute. 2016;353(15):3857-3875.

[24] Argha A, Li L, Su SW, Nguyen H. Stabilising the networked control systems involving actuation and measurement consecutive packet losses. IET Control Theory \& Applications. 2016;10(11):1269-1280.

[25] Argha A, Li L, Su SW. Sliding mode stabilisation of networked systems with consecutive data packet dropouts using only accessible information. International Journal of Systems Science. 2016;p. 1-10.

[26] Li L, Ugrinovskii VA, Orsi R. Decentralized robust control of uncertain Markov jump parameter systems via output feedback. Automatica. 2007;43:1932-1944.

[27] Niu Y, Lam J, Wang X, Ho DW. Observer-based sliding mode control for nonlinear state-delayed systems. International Journal of Systems Science. 2004;35(2):139-150.

[28] Khalil HK. Nonlinear Systems, 3rd Edition. New York: Prentice Hall; 2002.

[29] Grossmann IE. Review of nonlinear mixed-integer and disjunctive programming techniques. Optimization and Engineering. 2002;3(3):227-252.

[30] Löfberg J. YALMIP: A toolbox for modeling and optimization in MATLAB. In: CCA/ISIC/CACSD; 2004. Available from: http://control.ee.ethz.ch/index.cgi?action=details;id=2088; page=publications.

[31] Toh KC, Todd MJ, TÃijtÃijncÃij RH, Tutuncu RH. SDPT3 - a MATLAB software package for semidefinite programming. Optimization Methods and Software. 1998;11:545-581.

[32] Ogata K. Modern control engineering. Prentice-Hall Inc.; 1997. 